\title{
Processes and ICT Tools for ADHD Assessment, Intervention and Attention Training
}

\author{
http://dx.doi.org/10.3991/ijet.v9i6.4001 \\ Athanasios Drigas, Aglaia Tourimpampa \\ N.C.S.R. Demokritos, Athens, Greece
}

\begin{abstract}
This paper is intended to offer a comprehensive review on current research trends in the field of cognitive science and in particular, in the sector of the meta-cognitive attention skill. The paper features the latest developments in the research of attention and the cases in which attention is distracted due to ADHD (Attention Deficit Hyperactivity Disorder). More specifically, the review describes the certain types of attention such as selective, sustained, divided, focused and alternating attention and the attention function processes. Mainly, it focuses on thoroughly examining the methods and processes, as well as the ICT tools for ADHD Assessment, Intervention and Attention training. Finally it states the present achievements of the scientific research, it focuses on the impact of ICT as well as the role of parents and teachers in the confrontation of attention disabilities, it summarizes the standardized tools and rating scales of attention assessment, it goes in further examination of the association of attention with other meta cognitive skills, it points out questions that rise out of this examination, parts that need more intensive investigation, and estimates the future orientation of the attention research.
\end{abstract}

Index Terms-Attention, Types of Attention, Attention Functions, ICT Tools, ADHD, Assessment, Intervention, Training of Attention

\section{INTRODUCTION}

Attention is one of the most important skills that human has in order to accomplish his goals in his daily life. When he pays attention to a certain activity, he has the ability to solve problems of everyday life, he also presents a great improvement in his work or has makes a great progress in his studies. But the more attention is important in our lives, the easier can be distracted due to every day life's conditions. Especially in nowadays, there are lots of stimuli during our work, or when we are driving our car, or talking in the cell phone that can very easily distract our attention. These stimuli can be visual, auditory or perceptual.But why is crucial to study what happens when people lose their focus in what they do? What are the results when our attention is being distracted? Is it possible or not to maintain our attention focused all the time? What are the reasons which make inevitable the fact that we have to face this phenomenon? Which are the most common cases of attention distraction and which people have the tendency to lose focus of their attention? All the above, are matters that need to be investigated in order to make clear what the attention skill is. Mainly, attention is a skill that is being trained the moment we are born. Therefore, we have to study how attention is being developed at the first years of our lives and then focus on the conditions which affect people's attention skill. Attention includes different types such as Selective attention, sustained, multitasking and divided, focused and alternating one. Selective attention can be featured in visual and auditory one and is thought to operate as a two-stage process. Sustained attention is the ability of the person to hold his attention and not to be distracted. Focused attention refers to the ability to respond discretely to specific visual, auditory or tactile stimuli-Divided attention refers to the ability to respond simultaneously to multiple tasks or multiple task demandsAfter examining types of attention and its relation with other meta-cognitive skills, it is appropriate to refer that there certain processes with which attention functions. There is a certain way of the orienting of attention as well as the top down and bottom up processes of attention activation.[1]

Over the last years ADHD seems to occur more often in children and that is the reason why several attempts have been already made to approach the assessment, intervention of these disorders as well as attention training too. These efforts are based on behavior, symptoms, IQ, cognition, interview results, rating scales measurements, culture and other. In this review, we focus on the basis of some of them such as behavior -oriented processes and methods, as well as on ICT tools. Therefore, at this point we must refer to the great contribution and achievements of ICT on the field of ADHD. New technologies have promoted significantly ADHD research, as they provide a different aspect for these mental disorders, they have managed to systematize the investigation on this field, and they also provide more accurate and valid measurements as they are adapted to today's life of children with ADHD.

\section{Processes AND ICT TOOLs For AdHD} ASSESSMENT, INTERVENTION AND ATTENTION TRAINING

This review focuses on the attention disorders of children and adolescents between 10-18 years old and examines the conditions in which attention is being distracted. As a result, there are several occasions which are problematic as far as it concerns attention, and affect humans everyday life, their behavior, cognitive development of child, or cognitive procedure inside classroom and indeed, they need to be investigated. For this purpose, there are several ICT applications and systems for the ADHD Assessment, Intervention as well as for Attention Training.

\section{AdHD AsSESSMENT PROCESSES}

\section{A. Behavior Based}

Joseph Biederman et al. evaluated the common elements of CBCL (Child Behavior Check List) scales with the diagnosis of ADHD and mental disfunctions in 133 ADHD and 118 normal control boys, aged 6-17 years old. The comparison results showed that there is an absolute coincidence between the CBCL Attention problems Scale 
with the diagnosis of ADHD, between the delinquent Behavior Scale and the diagnosis of $\mathrm{CD}$, and between the Anxiety/Depression Scale and the diagnosis of Anxiety Disorders. These findings show that CBCL could be a useful tool for the assessment of comorbid and noncomorbid cases of ADHD.[2]

As following, in reference of Steven K. Reader, Lindsey M. Stewart, James H. Johnson, there is an attempt to make a further investigation on the results of the "Disruptive Behavior Stress Inventory" (DBSI) in order to measure the influence of stress factors on a child with ADHD. It was used a larger sample to point out the difference between primary caregivers of children with and without ADHD, according to the DBSI items. Most studies investigating ADHD caregivers stress levels also have used PSI which is a measure designed to access sources of strain to the parent-child relationship. The results tend to prove the adequacy of DBSI in the assessment of ADHD as far as concerns the relation between behavior-related stressors experienced by primary caregivers of children with ADHD. [3]

Isquith PK and Gioia GA, in order to understand the relationship between the construct of executive function and the diagnostic categorization of attentional disorders, the scientific research investigates a new measure of executive function for identifying children with likely ADHD subtypes. The Behavior Rating Inventory of Executive Function (BRIEF) is an 86-item questionnaire designed to assess executive functions using parents' and teacher observations on childrens' everyday behaviors. As predicted, the parents' notes confirm the clinical utility of BRIEF in indicating the distinction between ADHD subtypes, Combined-Type group and non- ADHD group.[4]

Another method proposed by Rosemary Flanagan is Behavior Assessment System for Children (BASC). It is multi-dimentional and multi-method of evaluating the behavior and self-ratings of children and adolescents aged 4-18 years. There are five components that can be used in any combination for a multi-method assessment: Parent rating, teacher rating and self-rating, developmental history and classroom observation. Moreover, it is important for BASC to mention the convergence with IDEA scale (Individuals with Disabilities on Education Act).[5]

Tom Manley et al. assumed that attention is not a separated mental process. For this reason, several different tasks are needed to assess different attentional disorders. Consequently, it is described the Test of Every Day Attention for Children (TEA-Ch) which includes nine subtests related to IQ, existing measures of attention and school achievement. This Test functions is developed on the basis of the belief that ADD is associated with poor selfsustained attention and behavioral control. [6]

\section{B. Symptom Based}

Thomas W. Frazier, Allison R. Frazier, Robin M. Busch, Melissa A. Kerwood and Health A. Demaree present how reliable is the study of symptoms which examine the resemblance of ADHD and RD. Scores from "Validity Indicator Profile (VIP) and the "Victoria Validity Symptom Score" ( VSVT) were the most valid at distinguishing simulating ADHD and RD. Percentages of control participants and participants in simulation conditions scoring below a specified cut score are provided to give clinicians an estimate of the simulator (true) positive and control (false) positive rates.[7]
Lindsey J. Jasinksi, Jordan P. Harp, Anne L. ShanderaOchsner, Lisa H. Mason and John D. Ranseen evaluate the use of symptom validity tests to detect college students who fake ADHD. The results of Test of Memory Malingering (TOMM), Letter Memory Test (LMT), Digit Memory Test (DMT), Nonverbal Medical Symptom Validity Test (NV-MSVT), and the b Test were remarkable at distinguishing feigned and truly existing ADHD. [8]

\section{IQ Based}

Macleod and Margot Prior describe the comparison of children with ADHD/H (Hyperactivity) to those without (ADHD/WO) as far as concerns the cognitive functioning. They were also selected 16 children from regular classroom for research purposes. Intelligence Test Scores ( Paced Auditory Serial Addition Test and the Letter Cancellation Task) present differences between the two ADD groups. Specifically, the ADD/H children attained significantly lower IQ scores than both $\mathrm{ADD} / \mathrm{WO}$ and control children. In general, the differences between the two groups were fewer than expected and more global than specific, on the basis of previous research.[9]

The same conclusion derives from reference of Henrik Larsson, Paul Lichtenstein and Jan-Olov Larsson in which is showed that the co-occurrence of ADHD and IQ scores has genetic origins. This examination is developed to study how genes are responsible for the symptoms of ADHD. The investigation was based on DSM criteria of ADHD and displayed a correlation between the generic factor and the cross-subtype or specific subtype ADHD according to the DSM categorization subtypes.[10]

\section{Culture Based}

Sami Timimi and Eric Taylor encounter ADHD as a cultural construct. According to this, some authorities suggest that ADHD is caused by the institutions of society. For example, school, or social cohesion, or leisure activity can be blamed for children going "out of control". Moreover, social factors influence the degree of hyperactivity that is seen as a problem. [11]

\section{E. Cognition Based}

Aaron P. G examined the degree of connection between Attention distraction (ADD) and Reading Disorder (RD). It is presented a model of comparative assessment of ADHD and RD. It is assumed that children with ADD would have low achievements in listening comprehension which require sustained attention than on tests such as reading comprehension which do not need so intensive attention. They were applied tests of reading comprehension (Woodcock, Cloze format) and Gates McGinitey (Paragragrah format). They were also applied the test of Listening comprehension from Woodcock Language Proficiency Battery as well as Woodcock listening and reading vocabulary. The validity of the model was tested by comparing the differential performance of the children on these tests with that of Conners Continuous Performance test (CPT) which measures attentional disorders. Considering the results of the above tests there has been a distinction between $\mathrm{ADD}$ and $\mathrm{RD}$ in a) reading and listening comprehension tests. b)Reading comprehension test in Cloze format and Reading comprehension test in Paragraph format c) Application of reading comprehension test in one session and in two sessions. [12] 


\section{ADHD ASSESSMENT ICT TOOLS}

\section{A. Behavior Based}

In reference of Ernest M. Post, Mitchel S. Burko and Michael Gordon, are presented data on standardization and reliability of a behaviour based measure of attention and self-control. It is used the Gordon Diagnostic System (GDS) , a micro-processor based portable device that administers a series of game-like tasks that measures the ability of child to sustain attention and inhibit behavioural responding. [13]

\section{B. Computer Based}

Joan C. Ballard presents a computerised assessment of sustained attention and reviews a series of factors that affect vigilance performance. These factors are distinguished in three categories: task parameters, environmental or situational factors and subject characteristics.[14]

Young jun Kim, Randal W. Hill and Jr. David R. Traum propose the development of a computational model of perceptual attention that administers top down and bottom up attention processes of virtual humans in virtual environments.[15]

Another effort for developing ICT tools for ADHD assessment is the review in reference of Shana L. Nichols, Daniel A. Waschbusch in order to check the validity of laboratory cognitive tasks used to assess symptoms of ADHD. These tasks are Continuous Performance Test (CPT), Gordon Diagnostic System (GDS), the Children's Checking Test (CCT), the Choice- Delay Task (C-DT) and Stop Signal Task. [16]

In a more specific view for assessing ADHD, in reference of Elisabeth H. Wiig, Stephanie S. Jones and Erik D. Wiig there is the Computer-based and standard administrations of the Test of Word Knowledge (TOWK)-Level 2 core subtests (Word Definitions, Synonyms, Figurative Usage, Multiple Contexts) (Wiig \& Secord, 1992), compared for 30 subjects with learning disabilities. Half completed the computer-based version first and half the standard version first. Three weeks later, subjects were given either the standard or computer-based version in a counterbalanced design. The total, receptive, and expressive composites and three subtest means were highest for the standard administration. It is implied form the above examination, that differences in task formats highlight the individual study for each computer based application in language tests. [17]

Moreover, another system in four studies is presented in reference of Cisero Cheryl A. et al., that evaluates the reliability of the computer-based academic assessment system (CAAS) as a diagnostic tool for detecting reading disabilities in college students. CAAS assesses component reading skills using computer-presented reading tasks that measure speed and accuracy of reading achievement. CAAS validity was carried out upon 4 requirements: The technique must (a) be valid for the purpose of detecting reading disability, (b) provide data that are compatible with theories of reading disability, (c) provide ad hoc elements of the student's problem, and (d) propose interventions of students' learning problems. Study results show that the CAAS system can satisfy all 4 criteria. [18]

\section{AdHD INTERVENTION PROCESSES AND ICT TOOLS}

\section{A. Computer Based}

Barbara K. Given, John D. Wasserman, Charmila A. Chari, Karen Beattie and Guinevere F. Eden presented the premise that computer based intervention that target auditory temporal processing combined with language exercises (Fast forWord) is effective in remediating children with disorders of language and reading. In sixty five struggling readers, were administered the following interventions: 1) two phases of intervention with Fast ForWord 2) two phases of intervention with Success Maker 3) FFW followed with SM 4) SM followed with FFW 5) no other intervention expect for the class curriculum. Results indicated significant within subjects effects, but no between subject groups differences.[19]

In the present reference, David L Rabiner, Desirre W. Murray, Ann T Skinner, Patrick S. Malone and J Abnorm evaluated the impact of Computerized Attention Training (CAT) and Computer Assisted Instruction (CAI) on attention and academic performance in 77 inattentive first graders. Results provide initial evidence that CAT and CAI can improve children's attention in the classroom. [20]

The following investigation of Yeunjoo Lee and Cynthia O Vail, examines how children with developmental disabilities can perform better in word recognition using a computer program. The intervention program was developed through a formative evaluation process and it includes modalities such as videos, sounds, texts and animations. A further investigation of this program also evaluated the effects of CAI. Four word sets, replicated by four boys with developmental disabilities who participated, were used to evaluate the effectiveness of the program. A further investigation of this program also evaluated the effects of CAI. Findings indicate that all children acquired the target words. [21]

Veronica L Raggi and Andrea M. Chronis proposed interventions in academic impairments of children with ADHD using computer based methods. For instance, it is applied the Computer Assisted Instruction (CAI) which includes specific instructional objectives such as essential material, use of multiple sensory modalities, division of content into smaller chunks of information, provision of immediate feedback about response accuracy. This method has been suggested in order to improve children's with ADHD sustained attention and work performance. [22]

In study of Jeanne Ecalle, Annie Magnan and Caroline Calmus, are examined the effects of a computer-assisted learning (CAL) program in which uses word segmentation in syllabic units. They were studied two separate groups of French speaking poor readers $(2 * 14)$ in first grade by being matched on a range of reading measures. Three tasks were applied using a pre-test/training/post-test design, written word recognition, word reading aloud and word spelling. Furthermore, three post-test sessions were also administered: one just after training, one after 4 months, and a last one after 9 months. The experimental group trained with the CAL using syllabic units outperformed the control group using CAL with whole word recognition in all the three tasks and there were important lasting effects. It is important to mention that all the above can be tested with the assumption of self-teaching word recognition. [23] 


\section{Attention Training Processes And ICt ToOls}

\section{A. Training Processes}

A very remarkable effort by Norman W. Park, Guy B. Proulx and Wanda M. Towers, examines how successful is Attention Process Training (APT), in training different types of attention through a variety of tasks correlated to each type. The APT programme was administered to 23 traumatically brain-injured (TBI) participants. Results show that performance of the TBI participants improved after training on the primary outcome measures, but did not improve significantly more than the performance of a control group, given the outcome measures twice, but no training. As concluded, this program does not restores all the damaged attention functions, but does enhances learning of certain skills.[24]

Kimberly A. Kerns, Karen Eso and Jennifer Thomson discusse whether "Pay Attention!", an intervention training sustained, selective, alternating, and divided attention, could be utilized in a clinical setting with children diagnosed with ADHD, and whether children who received the intervention improved their attention and executive functioning. 23 school-aged children with ADHD participate in up to 16 sessions of "Pay Attention!" and the results are evaluated. Results show the intervention is feasible to administer and acceptable to participants. Parents and clinicians rate fewer ADHD symptoms following the intervention and report improvements in executive function. Child performance on neuropsychological tests showed improvements in reasoning, cognitive capability and working memory. The findings suggest the acceptability of "Pay Attention!" as a treatment for attention and executive functioning deficits in ADHD. [25]

Lilach Shalev, Yehoshua tsal and Carmel Mevorach presentthe Computerized Progressive Attentional Training (CPAT) program is composed of four sets of structured tasks which activate sustained, selective, executive attention and orienting attention. Twenty 6- to 13-yearold children with ADHD as the experimental group, followed the CPAT sessions twice a week over an 8-week period.

Sixteen age-matched control children with ADHD as the control group and participated in sessions of the same frequency, length, and format except that instead of performing the training tasks they played various computer games during the session. There were major achievements of the experimental participants in non trained measures of reading comprehension, and passage copying as well as a significant reduction of parents' reports of inattentiveness. No significant improvements were observed in the control group. Therefore, it can be implied that CPAT had a great impact in academic and attentional improvement of children. [26]

Walter Sturm, Klaus Willmes, Bernt Orgass and Wolfgang Hartje examined the validity computerised training programmes for alertness, vigilance as well as for selective and divided attention based on games. This program was applied in patients with damage. Each patient received consecutive training in the two most impaired of the four attention domains. Control tests were distributed on the basis of a standardised computerised attention test battery comprising tests for the four attention functions. The results after training were considerable in all domains. It is important to mention that not only does the application provides with a high degree of specific training ef- fects in individual cases, but also prevents from the decrease the performance after non-specific training of basic attention problems. The results are discussed under the consideration of the hierarchical organisation of attention functions.[27]

Amishi P. Jha, Jason Krompinger and Michael J. Baimeu assumed that mindfulness training may alter or improve specific types of attention. Therefore, they are investigated three attentional subsystems: alerting, orienting, and conflict monitoring. Two types of mindfulness training (MT) programs were examined, and participants received a behavioral testing before and after training. One training group consisted of individuals naive to mindfulness techniques, participated in an 8-week mindfulness-based stress reduction (MBSR) course and focused on the development of concentrative meditation skills. The other training group consisted of individuals experienced in concentrative meditation techniques and received a mindfulness retreat. The results of the comparative examination of the two groups, showed that mindfulness training may improve attention-related behavioral responses by reinforcing functioning of specific subcomponents of attention. Whereas participation in the MBSR course improved the ability to endogenously orient attention and retreat participation appeared to support the development of attentional skills, which improved exogenous alerting-related process. [28]

\section{B. Training ICT Tools}

Naomi J. Steiner, Radley Christopher Sheldrick, David Gotthelf and Ellen C. Perri examine the efficacy of 2 computer-based training systems to teach children with ADHD to attend more effectively. Forty-one children with ADHD participated in 2 sessionsl of neuro feedback (NF) or attention training through a standard computer format (SCF), either immediately or after a 6-month wait (waitlist control group). Parents, children, and teachers completed questionnaires pre- and post intervention. Reports of parents in the NF condition stated a significant change on Conners's Rating Scales-Revised (CRS-R) and Behavior Assessment Scales for Children (BASC) subscales, while in the SCF condition, they reported significant change on the CRS-R Inattention scale and ADHD index, the BASC Attention Problems Scale, and on the Behavioral Rating Inventory of Executive Functioning (BRIEF). This randomized control trial points out the effectiveness of computer-based interventions for ADHD and proposes the use of them at schools. [29]

A computer-assisted cognitive training program is presented in reference of William J.Burns and Doil D. Montgomery it was used to treat a 13-year-old Caucasian male with ADHD. The child received a cognitive training computer program, Captain's Log, for 35 sessions. Pre/post differences on the Conners Parent Rating Scale noted a decrease on all subscales. The scores on the Conners Teacher Rating Scale were less conclusive. A 7-month follow-up argued that most of the acquired attention skills were maintained, but at a slightly lower level.[30]

Lastly, The efficacy of computer-assisted attention and memory retraining was evaluated with 15 severely headinjured patients. On this basis, reference of Ronald Ruff, Robert Mahaffey, Jeremy Engel, Charles Farrow and David Cox 5 presents a raining with selected exercises adapted to the individual's needs were provided from the THINKable program for up to 20 hours in both the atten- 
tion and memory rehabilitation. The experimental design resulted multiple baseline procedures through a pre- and post-group comparison. Significant results were documented on the computerized tasks, psychometric measures and on patient and observer ratings of everyday behaviours of attention and memory. [31]

\section{DISCUSSION}

As it can be implied from the above review, ADHD is a multi dimentional phenomenon and it comprises a variety of factors that are able to cause attentional disfunctions. Considering all the scientific efforts for ADHD assessment and intervention, we can derive that there are different approaches for this aim based on various characteristics of the patients such as behavior ADHD symptoms, cultural characteristics, IQ, measures and results from interviews, questionnaires and rating scales. Moreover, DHD assessment tends to be associated with poor grades, poor reading and math standardized test scores, and increased grade retention. ADHD is also associated with increased use of school-based services, increased rates of detention and expulsion. But the most important to mark, is the positive impact of ICT in dealing with ADHD. Findings of ICT and ADHD investigation are very promising and due to the use of ICT tools, results become more accurate and measurable and in order to have a clearer aspect of what happens really in ADHD. Although there are standardized rating Scales for the assessment and intervention, such as DSM criteria, however, computerized intervention seems to be more targeted. To this aim, parent and teacher contribution as well as self-control of children with ADHD is crucial, without leaving out the investigation of college students and adults with ADHD. As it is referred above, ADHD is a multidimensional phenomenon that needs to be examined along with other cognitive abilities. Research has proved that there is a connection of Attention with the cognitive skills. A theoretical model is constructed that links inhibition to 4 executive neuropsychological functions that appear to depend on it for their effective execution: (a) working memory, (b) self-regulation of affect-motivation-arousal, (c) internalization of speech, and (d) reconstitution (behavioral analysis and synthesis) and associates ADHD with secondary impairments in the above executive abilities. Lastly, it is appropriate to stress out the significance of ICT in all the attention procedures reviewed in this article. Technology provides contemporary methods of investigating ADHD. Children's access to computers has increased very much during the last decade as well as the use of computers in classrooms. Thus, researchers and educators have already pointed out the effectiveness of them in dealing with ADHD. Current multimedia applications make easier human machine interaction in order to improve ADHD investigation. As a conclusion, the question, if assessment, intervention and training of Attention is really effective, can be a challenge for researchers to evaluate it, as the future research leans on several factors that cause ADHD.

\section{REFERENCES}

[1] http://wiki.org/wiki/Attention

[2] Joseph Biederman, Stephen V. Faraone, Alysa Doyle, Belinda Krifcker Lehman, liana Kraus, James Perrin, Ming T. Tsuang: "Convergence of the child Behavior Checklist with Structured Interview- based Psychiatric Diagnosis for ADHD Children with and without comorbidiry", Journal of Child Psychology and Psy- chiatry. Vol 34, No 7, pp. 1241-1251,1993 http://dx.doi.org/10.1111/j.1469-7610.1993.tb01785.x

[3] Steven K. Reader, Lindsey M. Stewart, James H. Johnson: "ADHD -related family stressors with the Disruptive Behavior Stress Inventory (DBSI): A replication and extension", Journal of Clinical Psychology Med Settings (2009) 16: 148160http://dx.doi.org/10.1007/s10880-008-9140-9

[4] Isquith PK, Gioia GA: "BRIEF predictions of ADHD: Clinical Utility of the behavioral Rating Inventory of Executive Function for detecting ADHD subtypes in children", Archives of Clinical Neuropsychology 15 (2000) 653-850 http://dx.doi.org/10.1093/ $\operatorname{arclin} / 15.8 .780 \mathrm{a}$

[5] Rosemary Flanagan: "A review of the behavior Assessment System for Children (BASC): Assessment consistent with the Requirements of Individuals with Disabilities Education Act (IDEA)", Journal of School Psychology, Vol 33, No 2, pp177186,1995 http://dx.doi.org/10.1016/0022-4405(95)00003-5

[6] Tom Manly, Ian Nimmo Smith, Peter Watson, Vichi Anderson, Anna Turner Ian H. Robertson: " The differential assessment of children's attention: the Test of Everyday Attention for Children" (TEA-Ch).Normative sample and ADHD performance", Journal of Child Psychology Psychiatry, Vol 42, No8, pp.1065-1081, 2001 http://dx.doi.org/10.1111/1469-7610.00806

[7] Thomas W. Frazier, Allison R Prazier, Robin M. Busch, Melissa A. Kerwood, health A. Demaree: "Detection of simulated ADHD and Reading Disorder (RD) using symptom validity measures", Arxhives of Clinical Neuropsyxhology 23 (2008) 501-509 http://dx.doi.org/10.1016/j.acn.2008.04.001

[8] Lindsay J. Jasinski, Jordan P. Harp, Anne L. Shandera-Ochsner, Lisa H. Mason, John D Ranseen: " Using symptom validity to detect malingered ADHD in college students", The clinical Neuropsuchologist, 2011, 25 (8), 1415-1428

[9] Douglas Macleod \& Margot Prior: " Deficits in adolescents with ADHD and other clinical groups" (1996), Child Neuropsychology, A journal on Normal and Abnormal Development in Childhood and Adolescence, 2:1, 1-10

[10] Henrik Larsson, Paul Lichtenstein, Jan Olov Larsson: “ Genetic contributions to the development of ADHD Subtypes from Childhood to Adolescence", Journal of the American Academy of Child \& Adolescent Psychiatry,Vol 45 Issue 8, August 2006,Pages 973$981 \mathrm{http} / / / \mathrm{dx}$.doi.org/10.1097/01.chi.0000222787.57100.d8

[11] Sami Timimi, Eric Taylor: " ADHD is best understood as a cultural construct", The British Journal of Psychiatry, 2004, 184:8-9 http://dx.doi.org/10.1192/bjp.184.1.8

[12] Aaron P.G. : "A cognitive tool for the differential diagnosis of dyslexia from Attention Deficit Disorder Predominantly inattentive type". Dept of Educational Psychology, Indiana State University, Terre Haute, Indiana ,USA

[13] Ernest M. Post, Mitchel S Burko. Michael Gordon: " Single Component microcomputer-driven assessment of attention", Behavior Research Methods, instruments and computers, 1990, 22 (3), 297-301 http://dx.doi.org/10.3758/BF03209819

[14] Joan C Ballard; Computerized assessment of sustained attention: A review of factors affecting vigilance performance", Journal of Clinical and experimental Neuropsychology, 18:6, 843-863

[15] Young jun Kim, Randal W Hill, Jr David R. Traum: “ A computational model of Dynamic Perceptual Attention for Virtual Humans", Institute for Creative Technologies, 13274 Fiji Way, Suite 600, Marina Del Rey, CA 90292

[16] Shana L. Nichols, Daniel A. Waschbusch: " A review of the validity of Laboratory Cognitive Tasks used to assess symptoms of ADHD”, Child Psychiatry and Human Development, Vol34 (4), Summer 2004

[17] Elisabeth H. Wiig, Stefanie S. Jones, Eric D. Wiig: "Computerbased Assessment of Word Knowledge (TOWK) in Teens with Learning Disabilities", Language, Speech and Hearing Services in Schools, January 1996, Vol27, 21-28 http://dx.doi.org/10.1044/ 0161-1461.2701.21

[18] Cicero, Cheryl A Royer, James M. Marchant III, Horace G. Jackson, Stanley J: " Can the computer based academic assessment system (CAAS) be used to diagnose reading disability in college students?", Journal of Educational Psychology, Vol 89(4), Dec 1997, 599-620 http://dx.doi.org/10.1037/0022-0663.89.4.599 
PAPER

Processes AND ICT TOOLS FOR ADHD ASSESSMENT, INTERVENTION AND ATTENTION TRAINING

[19] Barbara K. Given, John D. Wasserman, Charmila A Chari, Karen Beattie, Guinevere F. Eden: "A randomized controlled study of computer based intervention in middle school struggling reader", Brain and Language 106 (2008) 83-97 http://dx.doi.org/10.1016/ j.bandl.2007.12.001

[20] David L Rabiner, Desirre W. Murray, Ann T Skinner, Patrick S. Malone, J Abnorm: “ A randomized trial for two promising computer based interventions for students with attention difficulties", Child Psychol (2010) 38: 131-142

[21] Yeunjoo Lee, Cynthia O Vail: “ Computer Based reading instruction for young children with disabilities", Journal for Special Education Technology 20(1), WINTER 2005

[22] Veronica L. Raggi, Andrea M Chronis: "Interventios to address the Academic Impairment of children and adolescents with ADHD”, Clinical Child and Family Psychology Review Vol.9 No2 June 2006 http://dx.doi.org/10.1007/s10567-006-0006-0

[23] Jeanne Ecalle, Annie Magnan, Caroline Clamus: "Lasting effects on literacy skills with a computer assisted learning using syllabic units in low progress readers", Computers and Education 52 (2009), 554-561 http://dx.doi.org/10.1016/j.compedu.2008.10.010

[24] Norman W. Park, Guy B. Proulx, Wanda M. Towers: "Evaluation of the Attention process training programme", Neuropsychological Rehabilitation 19999 (2) 135-154 http://dx.doi.org/10.1080/ 713755595

[25] Kimberly A. Kerns, Karen Eso, Jennifer Thomson: "Investigation of a direct intervention for improving attention in young children with ADHD”, Develpmental Psychology 16:2 273-295

[26] Lilach Shalev, Yehoshua Tsal, Carmel Mevorach: "Computerized Progressive Attentionla Training Program (CPAT): Effective Direct Intervnetion for Children ADHD", Journal on Normal and Abnormal Development in Childhood and Adolescence, 13:4,282388

[27] Walter Sturm, Klaus Willmes, Bernt Orgass \& Wolfgang Hartje: “Do specific Attention Deficits Need Specific Training?", (1997), Neuropsychological Rehabilitation: An international Journal, 7:2, 81-103

[28] Amishi P. Jha, Jason Krompinger \& Michael J. Baimeu: "Mindfullness training modifies subsystems of attention", Cognitive, Af- fective \&Behavioral Neuroscience, 2007, 7 (2), 109-119 http://dx.doi.org/10.3758/CABN.7.2.109

[29] Naomi J. Steiner, Radley Christopher Sheldrick, David Gotthelf and Ellen C. Perrin: "Computer-Based Attention Training in the Schools for Children with Attention Deficit/Hyperactivity Disorder: A preliminary trial”, CLIN PEDIATR 2011 50: 615 originally published online 10 May 2011

[30] Dilnavaz B. Kotwal, William J. Burns, Doil D Montgomery: “ Computer -Assisted Cognitive Training for ADHD. A case Study", Behav. Modif. January 1996, Vol 20 no1 85-96 http://dx.doi.org/10.1177/01454455960201004

[31] Ronald Ruff, Robert Mahaffey, Jeremy Engel Charles Farrow, David Cox and Peter Karzmark: "Efficacy study on THINKable in the attention and memory retaining if traumatically head injured patients", 1994, Vol.8, No1, Pages 3-4

\section{AUTHORS}

Athanasios Drigas is a Senior Researcher at N.C.S.R. Demokritos. He is the Coordinator of Telecoms Lab and founder of Net Media Lab since 1996. From 1985 to 1999 he was the Operational manager of the Greek Academic network. He has been the Coordinator of Several International Projects, in the fields of ICTs, and e-services (elearning, e-psychology, e-government, e-inclusion, eculture etc). He has published more than 240 articles, 7 books, 25 educational CD-ROMs and several patents. He has been a member of several International committees for the design and coordination of Network and ICT activities and of international conferences and journals. (e-mail: dr@iit.demokritos.gr).

Aglaia Tourimpampa is with N.C.S.R. 'Demokritos', Institute of Informatics and Telecommunications, Telecoms Lab - Net Media Lab, Agia Paraskevi, 153 10, Athens, Greece (e-mail: lidatou@gmail.com).

Submitted 03 July 2014. Published as resubmitted by the authors 08 December 2014. 